\title{
Factors affecting bulblet growth of Lilium sp. in vitro and in vivo
}

\author{
Md. Saiful Islam ${ }^{1 *}$, Md. Zohurul Kadir Roni ${ }^{1}$, and Kazuhiko Shimasaki ${ }^{2}$ \\ ${ }^{1}$ Department of Plant Resource Production, Kochi University, Monobe B200, Nankoku, Kochi 783-8502, Japan \\ ${ }^{2}$ Faculty of Agricultural Sciences, Kochi University, Monobe B200, Nankoku, Kochi 783-8502, Japan
}

\author{
*Corresponding author: saiful1236@gmail.com
}

\begin{abstract}
The major problem with in vitro propagation of lilies is small bulblet size, and the initial size of bulblets not only strongly affects growth and morphogenesis rates but also the transition between various vegetative and reproductive phases during development after planting. Therefore, bulblet growth is the most important factor in understanding how lily bulblets grow in vitro and the role of starch converted from sucrose in the medium on the growth mechanism in lilies, with the aim of revealing the effective in vitro culture condition to enhance in vivo performance of lily bulblets. The results of this study show bulblet growth correlates with the use of starch granule reserves inside explant tissue and in the medium. The main factor determining the growth of bulblets in vitro is explant size due to internal storage of starch, which plays a vital role in bulblet growth irrespective of cultivar. Furthermore, internal storage of sucrose plays a vital role and influences regeneration and bulblet growth in both in vitro and in vivo cultures. Therefore, the size of the bulblets produced in vitro strongly affects performance after planting. There is a high correlation $\left(\mathrm{R}^{2}=0.9672\right)$ between bulb weight after the growth season and initial bulb weight and therefore, the ontogenetic age of bulblets at planting. Hence, culturing lily scale explants in a high sucrose concentration during regeneration of bulblets in vitro to increase bulblet size is the main point of interest for future production.
\end{abstract}

Keywords: Lily, Sucrose concentration, Bulblet growth, Bulblet size, in vitro, in vivo.

\section{Introduction}

Lilies are among the top cut flowers in the world. Maintaining their genetic purity is required for commercially grown cultivars, so they are usually propagated by vegetative means. In vitro propagation technology for lily bulblet regeneration is the method used by commercial growers to produce large numbers of high-quality and disease-free plantlets in a short period of time (George et al., 2008). There are two important aspects of this propagation technology. First, the growth of regenerated bulblets is affected by the size of the explant. According to Langens-Gerrits et al. (2003b), "the larger the explants, the larger the bulblets that are regenerated". Second, sucrose is considered the best carbon source for in vitro tissue culture (Cheesman et al., 2010). It is usually partially or completely hydrolyzed in the medium into monosaccharides, glucose, and fructose, which are taken up by the plant tissues. The concentration of sucrose greatly affects the size of lily bulblets regenerated in vitro (De Klerk, 2012).

Regardless of the advantages of in vitro propagation of lilies, the major problem is small bulblet size (Kumar et al., 2001). The initial size of the bulblets produced in vitro strongly affects growth and morphogenesis rates but also the transition between various vegetative and reproductive phases during development after planting. Lily bulbs of different grades differ widely in their relative growth potential and productivity in the field or greenhouse because growth after planting is determined by the initial bulb weight (Kumar et al., 2001; Langens-Gerrits et al., 1996b). Hence, the growth of bulblets that are being produced is crucial. However, there are few studies available that clarify the growth of lily bulbs as a model for bulbous crops (De Klerk,
2009; Lian et al., 2003; Langens-Gerrits et al., 2003b; Langens-Gerrits et al., 1996b; De Klerk et al., 1992). Specifically, there are relatively few studies on a) the effect of different sucrose concentrations on bulb formation and the growth of bulbous plants and b) the effect of tissue culture bulblets on season performance in soil. A clear understanding of the factors affecting bulblet growth of lilies in vitro and in vivo would help to improve the optimal growth process. Hence, the aim of this study is to reveal the effective in vitro culture condition to enhance in vivo performance of lily bulblets.

\section{Results \\ In vitro:}

\section{Establishment of aseptic cultures and regeneration \%}

In vitro differentiation of bulblets from bulb scale explants was examined in three cultivars of Lilium speciosum (Stargazer, Casablanca, and Cesare) grown on MS medium supplemented with $0,1,3,6$, and $9 \%$ sucrose for 90 days, and the results are shown in Figure 1. Bulblets regenerating on scale segments or explants were categorized into two groups in this study depending on their size and position on the explant: full-scale and basal explants. The regeneration \% was significantly well, so bulblets were formed from the bulb scales. New bulblets from bulb scales produced by tissue culture formed in correlation with sucrose concentrations. The results show the effect of sucrose concentration on the regeneration $\%$ of each cultivar (Figure 1). Different concentrations of sucrose in the medium had a significant 
correlation to the differentiation of bulblets from bulb scale segments in vitro.

\section{Effect of sucrose concentration on days to regeneration}

The influence of different sucrose concentrations $(0,1,3,6$, and $9 \%$ ) was investigated by comparing the days to regeneration in vitro. Figure 2 shows that both scale segments or explants exhibit similar patterns of time to regeneration irrespective of Lilium cultivar. Sucrose concentrations of 3 and $6 \%$ resulted in minimum days to regeneration, with $1 \%$ for maximum days. Hence, sucrose concentration significantly affects days to regeneration in vitro and might affect bulblet formation.

\section{Effect of sucrose concentration on bulblet fresh weight}

The effect of sucrose concentration on bulblet fresh weight was studied in three cultivars of $L$. speciosum (Stargazer, Casablanca, and Cesare) grown on MS medium supplemented with $0,1,3,6$, and $9 \%$ sucrose for 90 days. Sucrose may affect growth mainly during regeneration, especially during the induction period regardless of cultivar. There was a linear relationship between bulblet fresh weight and sucrose concentration in both explants (full scale and basal): the higher the sucrose concentration the larger the bulblets and hence, the higher fresh weight. At all concentrations, the bulblets from the full-scale explants were significantly larger and had more fresh weight compared with the bulblets from the basal explants (Figure 3). Hence, the experiments reveal that explant size influenced bulblet size and fresh weight during the culture period. Bulblet fresh weight was also positively affected by sucrose concentration because sucrose is a major nutritional factor that influences bulblet growth.

\section{Effect of sucrose concentration on bulblet number}

The influence of explant size and sucrose concentration was shown in more bulblets being regenerated at high rather than low sucrose concentrations (Figure 4). The type of explant had no significant effect, even within cultivars (Figure 4). In this experiment, the explants from all three cultivars were exposed to various sucrose concentrations, and thus bulblet number was positively affected by sucrose concentration because sucrose is a major nutritional factor that affects growth during the period of induction of bulblets in vitro (Langens-Gerrits and Miller, 2003).

In vivo:

Days to sprout

After 90 days of culture in vitro, the bulblets were planted in pots and placed in a greenhouse with optimum environmental conditions and the data collected for various parameters is presented in Table 1. Days to sprout was significantly influenced by sucrose concentration. The quickest sprouting occured with bulbs cultured in a higher sucrose concentration in the medium and the slowest with no sucrose (Table 1), which also correlates with bulblet growth activity in vivo.

\section{Number and types of leaves}

The maximum number of leaves were regenerated at high rather than low sucrose concentrations (Table 1), and cultivar type had no significant effect. All sprouted bulbs had rosette- type leaves (Table 1), not the desired sprouts with a stem, due to the initial minute bulb weight.

Bulb weight after the season and ontogenetic development Typically, all bulbs increase in weight after the growth season (Table 1), and there is a high correlation $\left(R^{2}=0.9672\right.$, Figure 5) between bulb weight after the growth season and initial bulb weight at planting. The ontogenetic development of all bulbs was at the juvenile stage due to the rosette-type leaves, and not at the desired stage of adult vegetative bulbs (leaves with stem).

\section{Discussion}

The factors affecting the regeneration and growth of lily bulblets on scale explant tissue was studied in vitro. According to Ascough et al. (2008b), several factors affect the formation of bulblets in vitro: the genetic makeup of the plant, in vitro culture conditions, and supplements added to the tissue culture medium. According to Rodrigues-Falcon (2006), sucrose influences cell division at the stolon swelling and so the most important factor is storage organ formation (Podwyszynska, 2012). The main factors determining the regeneration of lily bulblets are explant size and sucrose concentration in the medium. Higher regeneration percentages and minimum days to regeneration were observed from full-scale explants compared with basal explants. Sucrose concentration significantly affects bulblet growth (Kumar et al., 2005) irrespective of cultivar (Figure $6)$. This finding indicates that internal storage and transport of sucrose plays a vital role and influences regeneration and bulblet growth. However, sucrose taken up from the medium is mainly stored in the explant tissues and used for regeneration and growth of bulblets (Langens-Gerrits et al., 2003b). In this study, bulblets on the full-scale explants were always larger than those on the basal explants, even with different sucrose concentrations (Figure 3). Bulblets regenerating from scale segments in vitro usually use two nutrient sources for growth: the explant reserve and the culture medium. Hence, this result suggests that stored starch components in large explant tissue that support bulb growth cannot be compensated for in the small explants by starch from the culture medium. We hypothesize that larger bulblet formation on full-scale explants is mainly due to reserve starch content than that on small basal explants. If elements other than starch influence bulblet growth, it is not reflected in the different cultivars in vitro.

After 90 days of culture in vitro, the bulblets were planted in pots and placed in a greenhouse with optimum environmental conditions. The data collected for various parameters in (Table 1) varied significantly between the bulblets in the different sucrose concentrations. Bulbs cultured in higher sucrose concentrations sprouted earlier than those in no sucrose. The size of the bulblets produced in vitro strongly affected performance after planting, which indicates that the initial bulb size (Figure 6) and the stored sucrose from the sucrose in the medium has a definite effect. Similarly, the larger bulblet formation on the full-scale explants is mainly due to the reserve starch content compared with the bulblets on the small basal explants in vitro. Hence, planting bulblets (in soil) produced in vitro has shown that small bulblets emerge and grow more slowly (Lian et al., 2003), and the growth after planting greatly depends on the initial bulb weight (Langens-Gerrits et al., 1996b) due to sucrose storage in the bulblets. In addition, sprouting type is directly related to a switch in ontogenetic development from juvenile to adult vegetative (Figure 6). When bulblets are small (<300 mg), they sprout rosette-type of leaves instead of leaves with a 
Table 1. In vivo performance of lily bulbs cultured in vitro.

\begin{tabular}{|c|c|c|c|c|c|c|c|c|}
\hline Lily cultivars & $\begin{array}{c}\text { Sucrose } \\
\text { concentration } \\
(\%) \\
\end{array}$ & $\begin{array}{c}\text { Average } \\
\text { days to } \\
\text { sprout }\end{array}$ & $\begin{array}{l}\text { Number of } \\
\text { leaves }\end{array}$ & $\begin{array}{l}\text { Type of } \\
\text { leaves }\end{array}$ & $\begin{array}{c}\text { Initial bulb } \\
\text { weight } \\
\text { (mg) } \\
\end{array}$ & $\begin{array}{l}\text { Bulb weight after } \\
\text { the growth } \\
\text { season }(\mathrm{mg}) \\
\end{array}$ & $\begin{array}{l}\text { Ontogenetic } \\
\text { development }\end{array}$ & Remark \\
\hline \multirow{5}{*}{ Stargazer } & 0 & 38 & 4 & Rosette & 21.4 & 38.1 & Juvenile & \multirow{15}{*}{$\begin{array}{l}\text { No adult vegetative } \\
\text { bulb (all rosette type } \\
\text { leaves, no stem } \\
\text { sprouted) }\end{array}$} \\
\hline & 1 & 31 & 4 & Rosette & 56.0 & 70.1 & Juvenile & \\
\hline & 3 & 28 & 6 & Rosette & 70.2 & 86.4 & Juvenile & \\
\hline & 6 & 25 & 7 & Rosette & 75.0 & 89.9 & Juvenile & \\
\hline & 9 & 28 & 6 & Rosette & 72.0 & 91.4 & Juvenile & \\
\hline \multirow{5}{*}{ Casablanca } & 0 & 33 & 4 & Rosette & 25.0 & 35.0 & Juvenile & \\
\hline & 1 & 33 & 4 & Rosette & 57.0 & 73.4 & Juvenile & \\
\hline & 3 & 30 & 6 & Rosette & 68.0 & 85.5 & Juvenile & \\
\hline & 6 & 26 & 7 & Rosette & 75.0 & 96.0 & Juvenile & \\
\hline & 9 & 26 & 7 & Rosette & 71.0 & 87.3 & Juvenile & \\
\hline \multirow{5}{*}{ Cesare } & 0 & 31 & 4 & Rosette & 28.0 & 39.6 & Juvenile & \\
\hline & 1 & 29 & 4 & Rosette & 66.0 & 80.0 & Juvenile & \\
\hline & 3 & 24 & 7 & Rosette & 78.0 & 97.2 & Juvenile & \\
\hline & 6 & 24 & 7 & Rosette & 76.0 & 101.6 & Juvenile & \\
\hline & 9 & 25 & 7 & Rosette & 77.0 & 98.1 & Juvenile & \\
\hline
\end{tabular}

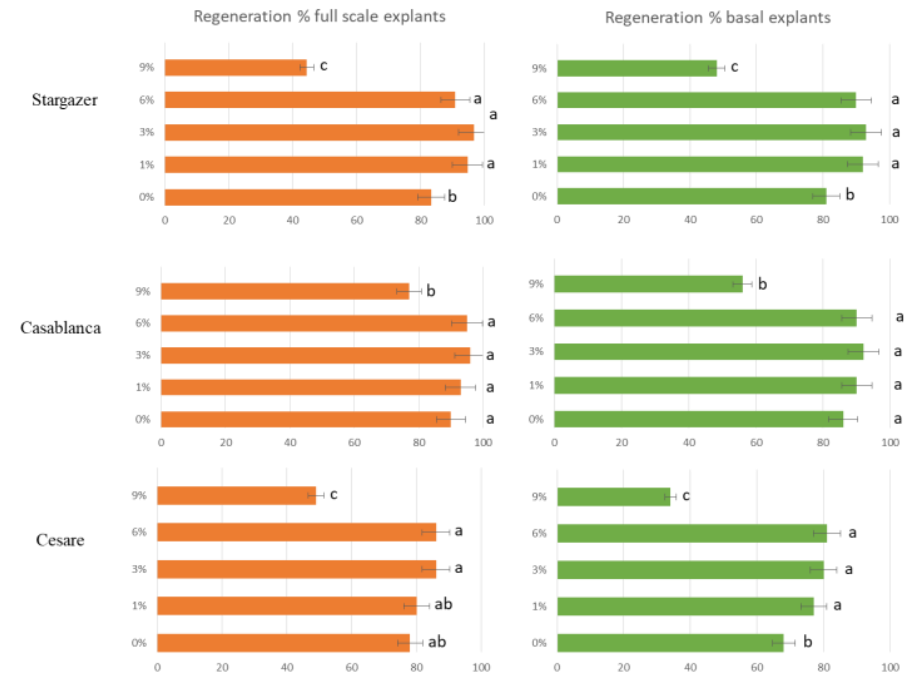

Fig 1. Effect of sucrose concentration on the regeneration \% in vitro. (Explants were cultured in vitro for 90 days under standard conditions and the number of sprouted explants was determined. Regeneration was calculated as a percentage of the surviving explants.).

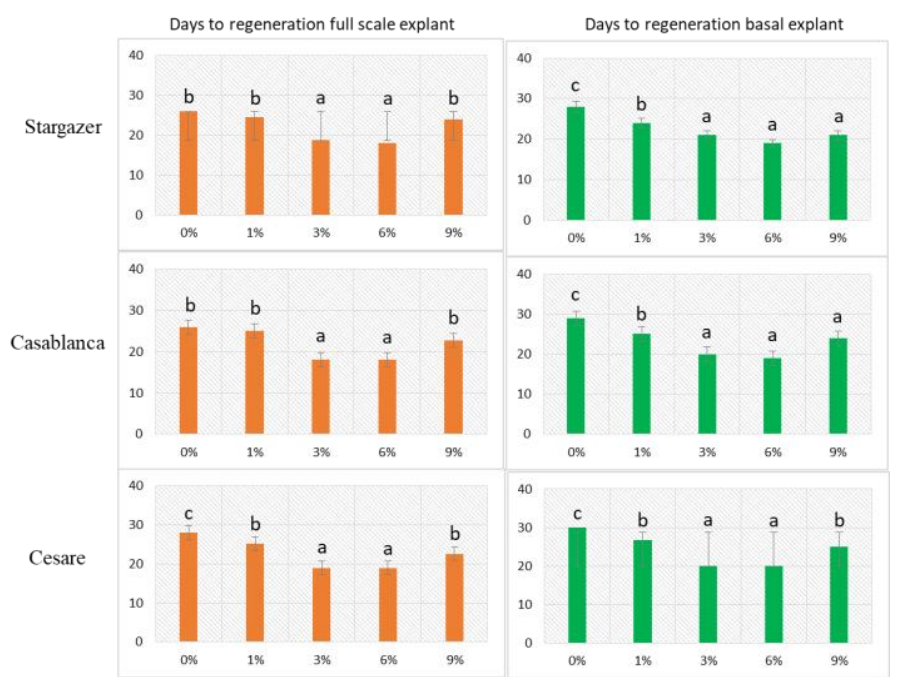

Fig 2. Effect of sucrose concentration on days to regeneration in vitro. (Sucrose concentrations on $\mathrm{X}$-axis and days to regeneration on Y-axis). 


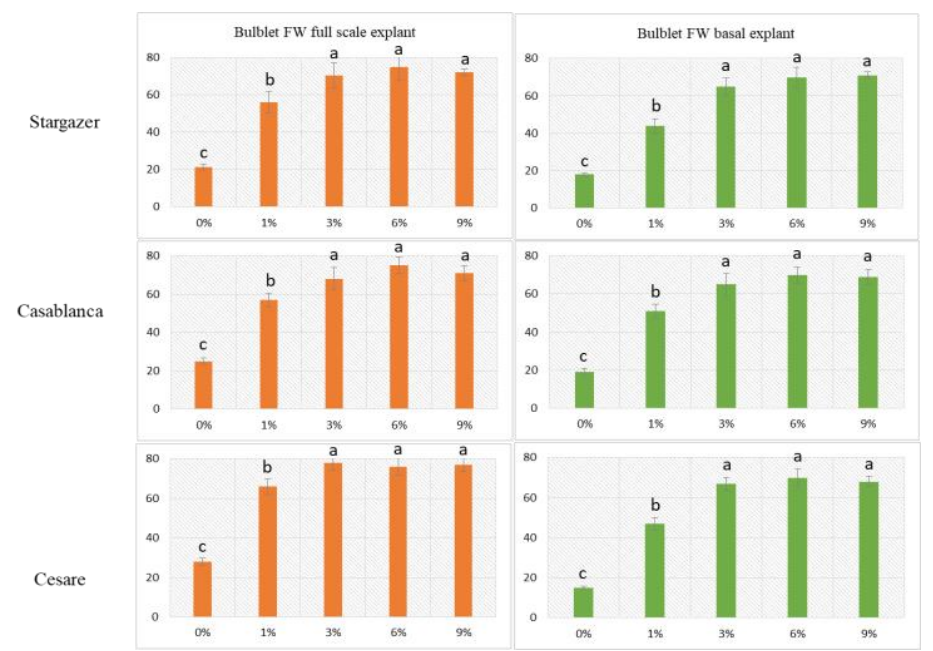

Fig 3. Effect of sucrose concentration on bulblet fresh weight (FW) in vitro. (Explants were cultured in vitro for 90 days under standard conditions and the fresh weight of the bulblets was determined. Each bar represents the average fresh weight of bulblets \pm SE.).

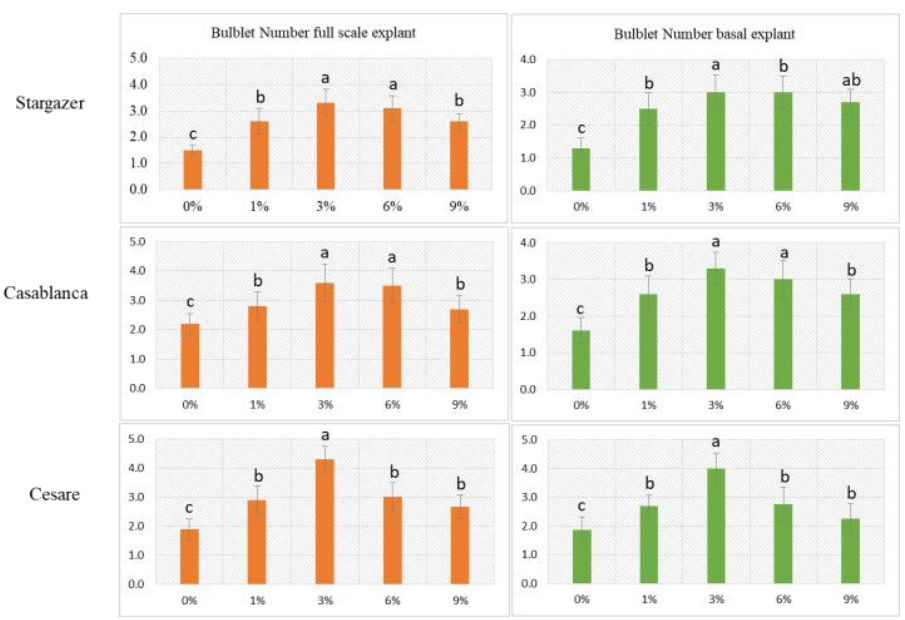

Fig 4. Influence of sucrose concentration on bulblet number. (Explants were cultured for 90 days under standard conditions and the number of bulblets was determined. Each bar represents the average number of bulblets \pm SE.).

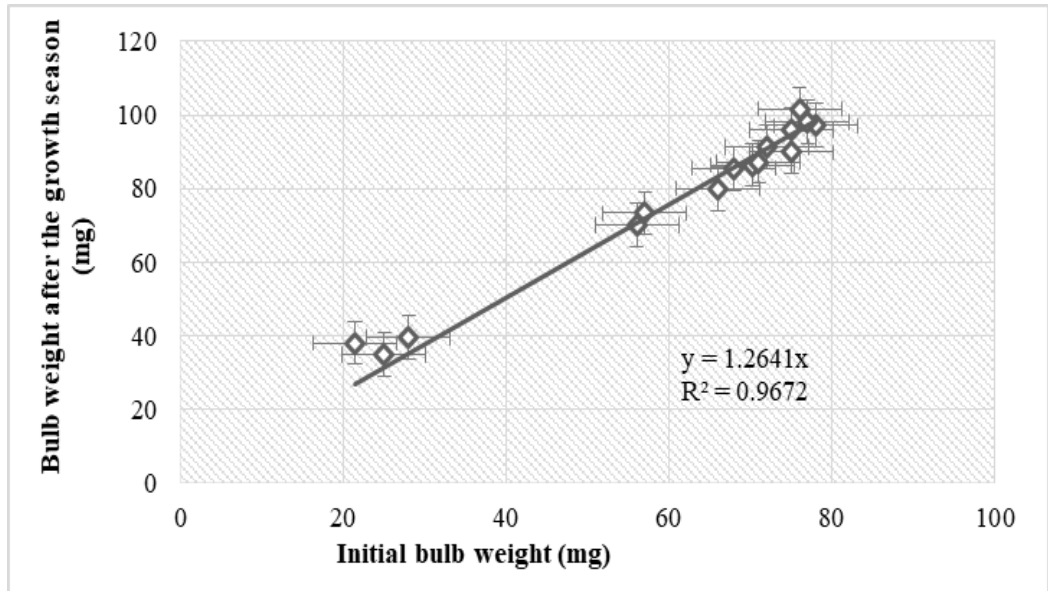

Fig 5. Correlation between bulb weight after the growth season and initial bulb weight at planting. 


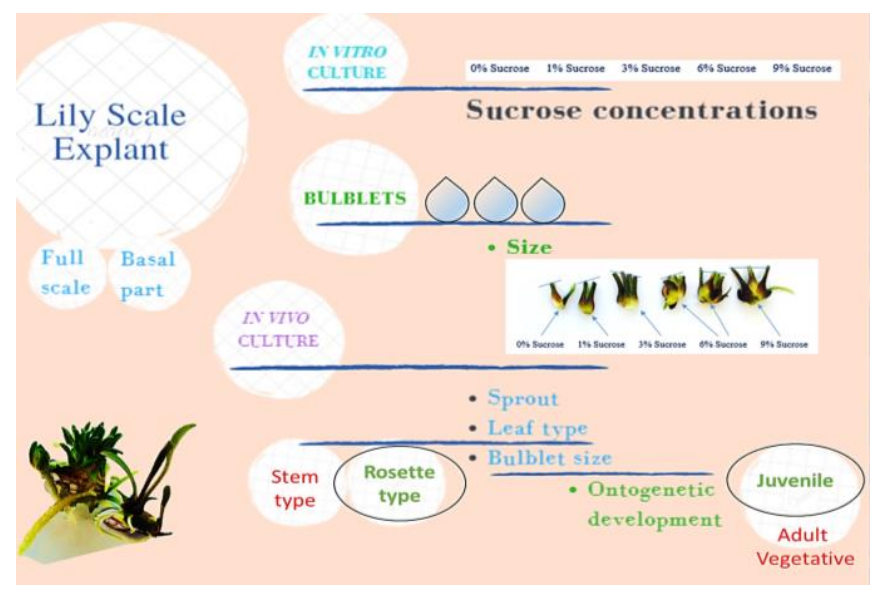

Fig 6. Factors affecting bulblet growth of lilies in vitro and in vivo.
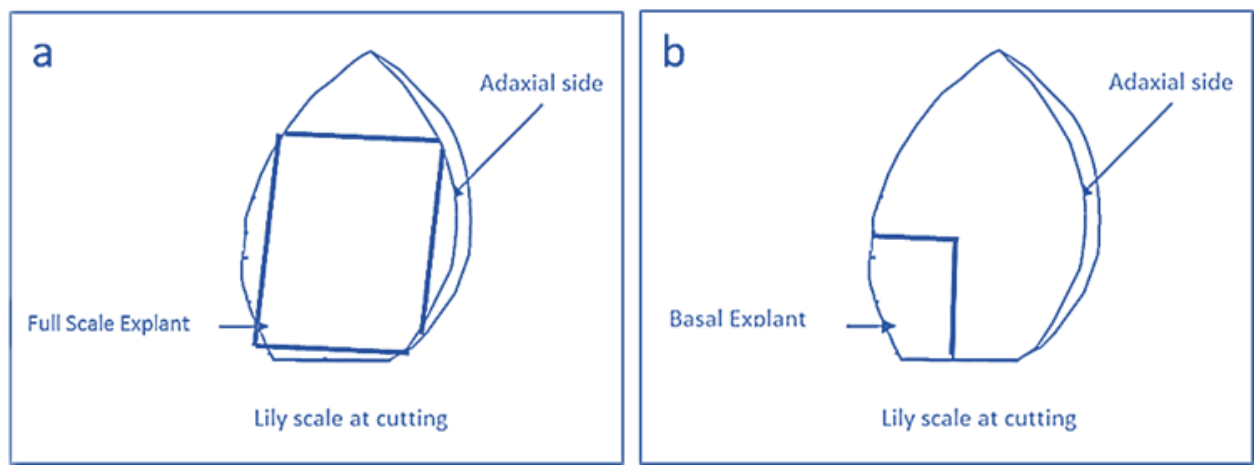

Fig 7. Schematic drawing of explants cut from lily scales: a) full-scale explant and b) basal explant.

stem (Langens-Gerrits et al., 2003a), so there was no adult vegetative bulb in this study. Therefore, the results from this in vivo study indicate that increasing bulblet size during in vitro culture is the main point of interest for future production.

\section{Materials and methods}

\section{Plant materials}

The lily cultivars (Stargazer, Casablanca, and Cesare) used in the in vitro experiment were collected from Nakamura-Noen Co., Ltd., Kochi, Japan. Full explants and basal (two different sizes) explants were used during bulblet regeneration in vitro. A schematic drawing of explants cut from lily scales is shown in Figure 7. The standard micro propagation protocol of lily was adapted from De Klerk (2012) in this study. Clean and healthy scales were rinsed in $70 \%$ ethanol, sterilized in $1 \%(\mathrm{w} / \mathrm{v}) \mathrm{NaOCl}$ for $30 \mathrm{~min}$, and rinsed three times in sterile de-ionized water. Bulblets were regenerated from scale explants with the abaxial side on $20 \mathrm{~mL}$ per explant of MSmedium. Sucrose is the most common carbon source used in plant cells, tissues, and organ cultures. Media with different sucrose concentrations have been used as a staple source since Murashige and Skoog (1962) described their MS medium.

\section{Medium preparation}

Medium was prepared using $4.4 \mathrm{~g} / \mathrm{L}$ MS medium with vitamins, NAA $50 \mu \mathrm{M} / \mathrm{L}$, and $7 \mathrm{~g} / \mathrm{L}$ micro-agar with sucrose
(0\%, no sucrose; $1 \%, 10 \mathrm{~g} / \mathrm{L} ; 3 \%, 30 \mathrm{~g} / \mathrm{L} ; 6 \%, 60 \mathrm{~g} / \mathrm{L}$; and $9 \%, 90 \mathrm{~g} / \mathrm{L}$ ) at $\mathrm{pH} 5.8$ adjusted prior to autoclaving for 15 min.

\section{In vivo plant material and culture condition}

After 90 days of culture in vitro, 10 morphologically uniform bulblets with similar initial weight were collected from each cultivar (Stargazer, Casablanca, and Cesare) and sucrose concentration and planted in pots $(9 \mathrm{~cm}$ in diameter and 15 $\mathrm{cm}$ in depth) containing fertilized soil with 380,290 , and 340 mg L ${ }^{-1}$ of N:P:K (Tanekura No. 42; Sumirin Agricultural Industry Co., Ltd., Japan). The pots were then placed in a greenhouse with optimum environmental conditions on $26^{\text {th }}$ February, 2016. After the full growth season, the bulblets were uprooted on $30^{\text {th }}$ September, 2016, and data were collected for various parameters.

\section{Statistical analysis}

The treatments were distributed in a completely randomized manner with 60 replications for each cultivar and sucrose concentration. The results are expressed as mean \pm standard error (SE). For all comparisons, statistical analysis was performed using one-way ANOVA, and subsequently Tukey's HSD test, with $p<0.05$ being considered statistically significant.

\section{Conclusion}

In conclusion, the main factors determining the growth of bulblets in vitro are explant size and sucrose concentration in the medium. Furthermore, internal storage and transport of 
sucrose plays a vital role and influences regeneration and bulblet growth. The size of the bulblets produced in vitro strongly affects performance after planting. From a horticultural point of view, we recommend culturing lily scale explants in a high sucrose concentration during regeneration of bulblets in vitro to increase bulblet size, which is the main point of interest for future production.

\section{Acknowledgements}

We thank the Laboratory of Floriculture and Vegetable Science, Kochi University, Japan for research support and also the Japanese Government for providing a scholarship (Monbukagakusho: MEXT) to MS Islam. We also thank Nakamura-Noen Co., Ltd., Kochi, Japan for providing the healthy bulbs and technical support during the in vivo culturing.

\section{References}

Ascough GD, Erwin JE, Van Staden J (2008b) In vitro storage organ formation of ornamental geophytes. Hortic Rev. 34: 417-445.

Cheesman L, Finnie JF, Van Staden J (2010) Eucomis zambesiaca baker: factors affecting in vitro bulblet induction. South Afr J Bot. 76: 543-549.

De Klerk GJ (2009) A cold treatment promotes both sprouting and sink strength of lily bulblets. Propagation Ornamental Plants. 9: 102-106.

De Klerk GJ (2012) Micropropagation of bulbous crops: technology and present state. Floriculture Ornamental Biotech. 6: 1-8.

De Klerk GJ, Kim K, Schadewijk MV, Langens-Gerrits M (1992) Growth of bulblets of Lilium speciosum in vitro and in soil. Acta Horticulture. 325: 513-520.
George EF, Hall MA, De Klerk GJ (2008) Plant propagation by tissue culture: volume 1 . The Background. Springer, 520pp.

Kumar S, Kashyap M, Sharma DR (2005) In vitro regeneration and bulblet growth from lily bulbscale explants as affected by retardants, sucrose and irradiance. Biol Plant. 49(4): 629-632.

Kumar S, Sharma DR, Sharma YD, Pathania NS (2001) In vitro propagation of Asiatic hybrid lily from bulb scales. Indian J Agr Sci. 71: 463-465.

Langens-Gerrits M, De Klerk GJ, Croes A (2003a) Phase change in lily bulblets regenerated in vitro. Physiol Plant. 119: 590-597.

Langens-Gerrits M, Kuijpers AM, De Klerk GJ, Croes A (2003b) Contribution of explant carbohydrate reserves and sucrose in the medium to bulb growth of lily regenerated on scale segments in vitro. Physiol Plant. 117:245-255.

Langens-Gerrits M, Lilien-Kipnis H, Croes T, Miller WB, Kollöffel C, De Klerk GJ (1996b) Bulb growth in lily regenerated in vitro. Acta Horticulture. 430: 267-274.

Langens-Gerrits M, Miller W (2003) Effect of low temperature on dormancy breaking and growth after planting in lily bulblets regenerated in vitro. Plant Growth Regul. 40: 267-275.

Lian ML, Chakrabarty D, Paek KY (2003) Bulblet formation from bulb scale segments of Lilium using bioreactor system. Biol Plant. 46: 199-203.

Murashige T, Skoog F (1962) A revised medium for rapid growth and bio assays with tobacco tissue cultures. Physiol Plant.15: 473-497.

Podwyszynska M (2012) The mechanisms of in vitro storage organ formation in ornamental geophytes. Floriculture Ornamental Biotech. 6: 9-23.

Rodrigues-Falcon M, Bou J, Prat S (2006) Seasonal control of tuberization in potato: conserved elements with the flowering response. Annu Rev Plant Biol. 57: 151-180. 